\title{
GABA Is Involved in Spatial Unmasking in the Frog Auditory Midbrain
}

\author{
Wen-Yu Lin and Albert S. Feng \\ Department of Molecular and Integrative Physiology and Beckman Institute, University of Illinois at Urbana-Champaign, Urbana, Illinois 61801
}

\begin{abstract}
Real-world listening situations comprise multiple auditory objects. Sounds originating from different objects are summated at the eardrum. The auditory system therefore must segregate the streams of sounds associated with the different objects. One listening strategy in complex environments is to attend to signals originating from one spatial location. In doing so, signal detection is compromised when a masker is present at close proximity, and detection is improved if the masker is spatially separated from the signal. A recent study has shown that, in frogs, spatial unmasking is more robust at the midbrain than at the periphery, indicating the importance of central mechanisms for this process. In this study, we investigated spatial unmasking patterns of single neurons in the frog inferior colliculus (IC) before and during iontophoretic application of bicuculline, a $\mathrm{GABA}_{\mathrm{A}}$ receptor antagonist. We found that drug application markedly decreased the strength of spatial unmasking such that even large angular separation of signal and masker sources produced only a weak masking release. Under the drug, the strength of spatial unmasking of midbrain neurons approximated that of auditory nerve fibers. These data show that GABAergic interactions in the auditory midbrain play an important role in spatial unmasking. Analysis of the effect of the drug on the direction sensitivity of the units shows that for the majority of IC units, bicuculline degrades binaural processing involved in directional coding, thereby compromising spatial unmasking. For other IC units, however, the decline in the strength of spatial unmasking is attributable to the effects of bicuculline on different central auditory processes.
\end{abstract}

Key words: inferior colliculus; torus semicircularis; stream segregation; release from masking; spatial unmasking; bicuculline; hearing; masking; signal detection

\section{Introduction}

Frogs communicate by sound in acoustically cluttered environments. Male frogs emit advertisement calls to attract females around a breeding pond that is often shared with numerous conspecific as well as sympatric males. It is generally up to the females to localize and discriminate the calls of conspecific males from calls of sympatric males to avoid interspecies breeding (Gerhardt, 1988). Despite the complexity in the calling environments, females show a high degree of success in call identification and localization. Within a chorus, conspecific males interact extensively such that their calls are conspicuous to the females (Wells, 1988). Males either emit calls alternately with other conspecific males to minimize call overlap or emit calls at higher rates. Furthermore, when another male intrudes on, or calls in close proximity to, a resident male, the latter elicits aggressive calls or engages in a physical fight to ward off the competition. Territorial interactions ensure that there is adequate spacing between males. Angular separation of signal and interfering sound sources produces a release from masking and improves the female's ability to localize and discriminate sound (Schwartz and Gerhardt, 1995).

\footnotetext{
Received April 10, 2003; revised June 23, 2003; accepted June 30, 2003.

This work was supported by National Institute on Deafness and Other Communication Disorders Grant R01DC00663 from the National Institutes of Health. We thank Alexander Galazyuk, David Gooler, Mario Penna, and Rama Ratnam for commenting on previous versions of this manuscript.

Correspondence should be addressed to Dr. Wen-Yu Lin, Beckman Institute, University of Illinois, Urbana, IL 61801. E-mail:w-lin5@uiuc.edu.

Copyright $\odot 2003$ Society for Neuroscience $\quad$ 0270-6474/03/238143-09\$15.00/0
}

Release from masking by means of source separation, or spatial unmasking, is also evidenced in humans (Bronkhorst and Plomp, 1988; Saberi et al., 1991; Gilkey and Good, 1995), ferrets (Hine et al., 1994), and birds (Dent et al., 1997).

The goal of this study was to advance our understanding of the central mechanisms responsible for spatial unmasking. Lin and Feng (2001) previously compared the spatial unmasking patterns of single neurons in the frog torus semicircularis (a homolog of the mammalian inferior colliculus) with those from the auditory nerve fibers. They reported that, in a free field, the strength of spatial unmasking is more robust in the inferior colliculus (IC) than at the periphery; the latter is weak but not negligible and is presumably attributed to acoustic shadowing of the head. Their results suggest that both the head-shadowing effect and central auditory processing contribute toward spatial unmasking, as suggested for humans (Yost, 1997). However, the central mechanisms responsible for spatial unmasking remain unclear.

The frog IC receives bilateral projections from the cochlear and superior olivary nuclei in the caudal brainstem (Feng and Lin, 1991). These brainstem nuclei themselves receive bilateral inputs and contain neurons having diverse binaural interaction patterns, with some exhibiting binaural facilitation and others showing binaural inhibition (Feng and Capranica, 1976, 1978; Zakon and Capranica, 1981). At present, it is unclear whether binaural facilitation, binaural inhibition, or both contribute to spatial unmasking. In the frog IC, the dominant binaural interaction pattern is binaural inhibition (Epping and Eggermont, 
1985; Gooler et al., 1996), and this is shaped in part by local GABAergic synaptic interactions (Zhang et al., 1999). In the present study, we tested the hypothesis that GABA-mediated inhibition was responsible for spatial unmasking evinced in the IC. For this, we evaluated the strength of spatial unmasking under two experimental conditions: before (and after) and during local application of bicuculline, a blocker of the $\mathrm{GABA}_{\mathrm{A}}$ receptor.

\section{Materials and Methods}

The general experimental methods were as described in an earlier paper (Lin and Feng, 2001). Experimental animals comprised 24 Northern leopard frogs (Rana pipiens) weighing 12-32 $\mathrm{gm}$. The animals were anesthetized by immersion in tricaine methanesulfonate (MS-222), and immobilized with an intramuscular injection of $d$-tubocurarine chloride $(10 \mu \mathrm{g} / \mathrm{gm}$ of body weight). A skin flap was incised on the dorsal surface of the head. Lidocaine (4\%) was applied topically to the wound area. A small opening was made in the skull above the optic tectum. After the surgery, the animal was placed in a soundproof chamber whose inner walls and ceiling were covered with 6" anechoic foams, and allowed to recover from anesthesia. Immobilization was maintained throughout the recording session by periodic injections of $d$-tubocurarine chloride. Each frog was used for one recording session of $8-10 \mathrm{hr}$. Afterward, the frog was killed by an overdose of MS-222. These procedures are in compliance with the Guiding Principles for Research Involving Animals and Human Beings and have been evaluated and approved by the Laboratory Animal Care and Use Committee of the University of Illinois.

Acoustic stimuli comprised tone bursts (duration, $200 \mathrm{msec}$; rise-fall time, $5 \mathrm{msec}$ ), broadband noise bursts [used as the masker $(\mathrm{M})$ ], and a prerecorded species mating trill [used as the probe $(\mathrm{P})$ ]. Tone bursts were used to acquire the general response properties of a unit. The waveforms and power spectral densities of the probe and the masker, as well as the power spectrum of the probe in the masker $(\mathrm{P}+\mathrm{M})$ are shown in Figure 1. The mating trill (Fig. 1D) was selected as the probe because it is the predominant call type in frog choruses; it has a duration of $900 \mathrm{msec}$ with energy concentrated over 150, 500, 700, 1000, and $1700 \mathrm{~Hz}$ (Fig. 1 A). Because of limitations of the data analysis software, we used only the first half of the trill as the probe (i.e., $450 \mathrm{msec}$ ); the two halves of the mating trill had the same trill rate, and they differed only in the envelope of the call. The masker (Fig. $1 E$ ) was a broadband noise with a flat power spectrum over the frog's audible range of $100-3000 \mathrm{~Hz}$ (Fig. $1 B$ ). The probe and masker were both digitized such that when they were presented through the digitalto-analog (D/A) converters (see below), they had a consistent temporal relationship. Frozen noise bursts were preferred over free-running noise bursts; the latter are undesirable because they have transient fluxes of energy in different frequency bands that vary from one trial to the next and thus can themselves elicit a change in the response of a unit. The duration of the masker was $450 \mathrm{msec}$, matching that of the probe; thus, $\mathrm{P}$ and $\mathrm{M}$ overlapped in time completely. Although temporally overlapped, at each sound level.
(B) Masker (M) @ $69 \mathrm{~dB}$ SPL

(C) P + M @ SNR of -6 dB
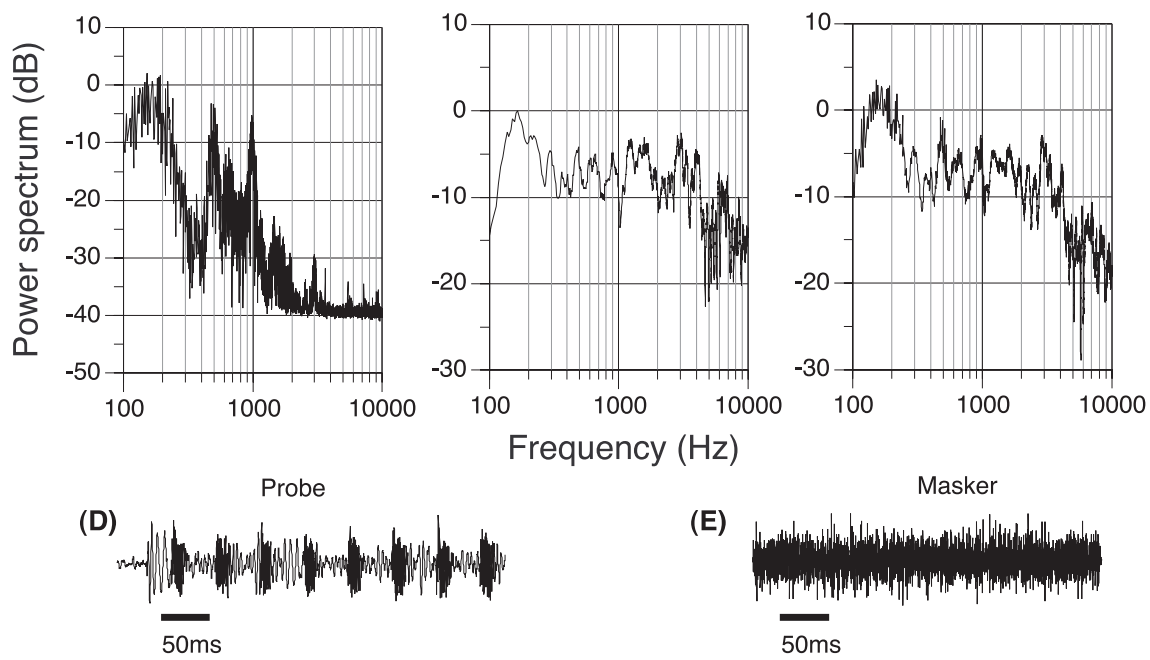

(E)

Masker

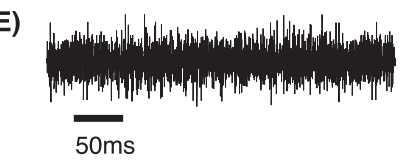

Figure 1. Mating trills of Rana pipiens (or the probe) and the masker used in this study. A, Power spectrum of the probe at 63 $\mathrm{dB}$ SPL. B, Power spectrum of masker at $69 \mathrm{~dB}$ SPL. C, Power spectrum of probe and masker at respective levels, i.e., at a signal-to-noise ratio of $-6 \mathrm{~dB}$. D , Waveform of the probe in its digitized form. $E$, Waveform of the masker in its digitized form.
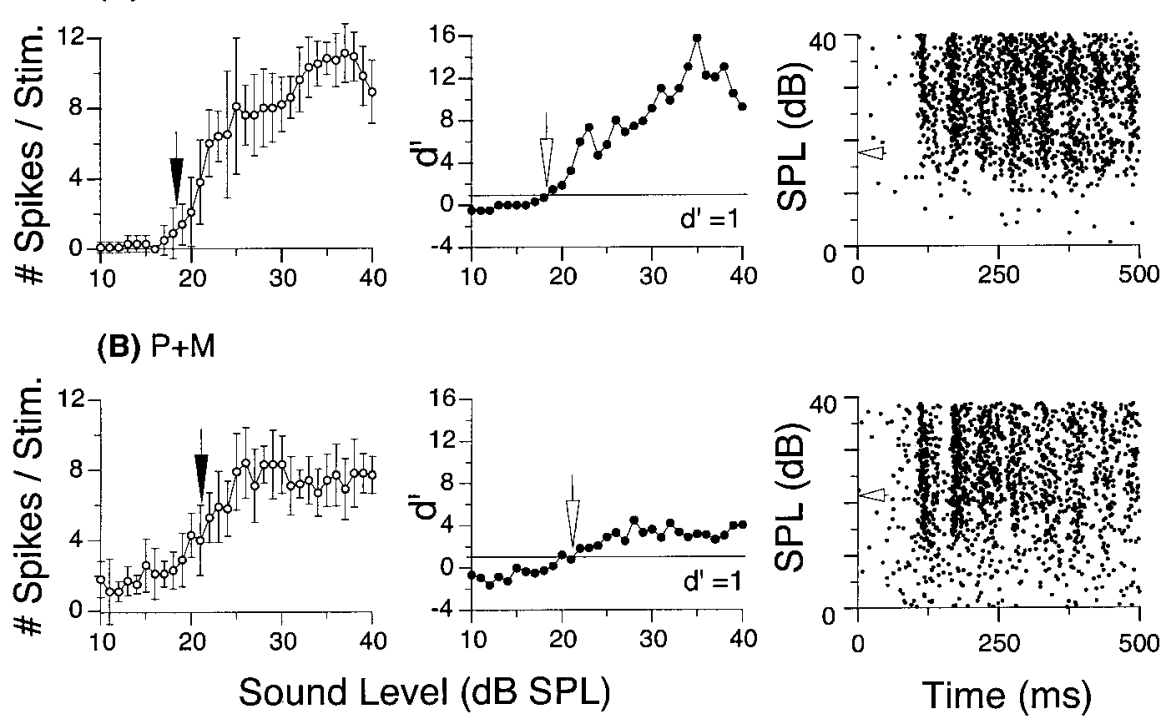

Figure 2. Rate level functions (left plots), $d^{\prime}$ functions (middle plots), and composite dot histograms (right plots) of an IC unit to $P$ alone $(A)$ and $P+M(B)$. In the left plots, the response of the unit at each sound level is shown by the average spike count \pm SD to 10 stimulus (Stim.) epochs. In the middle plots, the $y$-axis represents $d$ ' (for calculation, see Materials and Methods), and the open arrows indicate the probe detection thresholds to $P$ alone (18.3 dB SPL) and $P+M(21.2 \mathrm{~dB} \mathrm{SPL})$, namely, the sound levels at which $d^{\prime}=1$; the corresponding levels are shown by solid arrows in the left plots and by open arrowheads in the right plots. The composite dot histograms in the right plots show the timing of trains of action potentials (each spike is represented by a single dot) at various sound levels. Time 0 represents the onset time of the acoustic stimuli. In these plots, 10 traces of spike trains are shown could be distinguished from the response of a unit to the probe that typically showed time-locked firing patterns (Fig. 2). Time-locked firing was therefore a potential metric for estimation of probe detection (see below).

The stimulus parameters were controlled by Tucker Davis System 2 hardware and software from a personal computer. To present the prerecorded natural call and noise bursts, each digital file that was stored on the computer hard drive was retrieved and passed through a D/A converter (sampling frequency, $12 \mathrm{kHz}$ ), a programmable attenuator, an audio amplifier, and a loudspeaker.

$\mathrm{P}$ and $\mathrm{M}$ were broadcast through two separate loudspeakers (ADS 200 
LC) mounted on two separate hoops at $0^{\circ}$ elevation. These hoops could be rotated independently over a $180^{\circ}$ range in the frontal field using two separate mechanical systems (Lin and Feng, 2001). The frog was placed at the center of rotation of these hoops at a distance of $55 \mathrm{~cm}$ from the loudspeakers. The absolute sound pressure level (decibels with regard to $20 \mu \mathrm{Pa}$ ) was calibrated at the location of the frog's left tympanum using a free-field condenser microphone (Brüel and Kjaer 4165). Each loudspeaker was calibrated for each sound direction in the frontal field and equalized such that its frequency response was flat (within $\pm 3 \mathrm{~dB}$ over the frequency range of $120-3000 \mathrm{~Hz}$ and within $\pm 5 \mathrm{~dB}$ over $50-120$ and $3000-6000 \mathrm{~Hz})$.

Extracellular recordings were made from single neurons in the left IC using a single-barrel glass pipette (tip diameter, $1 \mu \mathrm{m}$; filled with $0.2 \mathrm{M}$ potassium acetate in $0.05 \mathrm{~m}$ Tris buffer) attached to a five-barrel glass pipette (tip diameter, $\sim 10 \mu \mathrm{m}$ ). Two of the five barrels were filled with bicuculline methiodide (BIC, 10 mM, pH 3.0; Sigma, St. Louis, MO), a $\mathrm{GABA}_{\mathrm{A}}$ receptor blocker. The central barrel was filled with $0.64 \%$ sodium chloride, $\mathrm{pH}$ 7.4, for maintaining electrical balance. The electrode assembly was attached to a piezoelectric microdrive and positioned stereotactically onto the dorsal surface of the optic tectum above the IC. The five-barrel pipette was connected to a six-channel iontophoresis current generator (Dagan 6400). A retaining current of $-15 \mathrm{nA}$ was used to prevent drug leakage at all times except during drug injection. Action potentials as picked up by the single-barrel glass pipette were amplified $10,000 \times$ by a preamplifier (Dagan 2400), filtered (passband of $100-6000$ $\mathrm{Hz}$ ), monitored audiovisually, and processed using Tucker Davis data acquisition software. The time of occurrence of action potentials was recorded using Brain-Ware, along with the waveform of action potentials. Times of spikes were registered by the software and used to construct the dot histogram off-line (Fig. 2, left plots). In the rare cases in which the electrode isolated more than one unit, responses from the individual units could be segregated on the basis of differential action potential waveforms. The recorded spike waveform was also used for identification of units during the long recording session (1.5-3 hr).

To search for auditory neurons, a series of five sequential tone bursts with five different carrier frequencies were presented at $90 \mathrm{~dB}$ sound pressure level (SPL) from the right $90^{\circ}$ [corresponding to contralateral $90^{\circ}\left(\mathrm{c} 90^{\circ}\right)$ ]; this represents the best azimuth for most units in the left IC (Gooler et al., 1996). All acoustic stimuli were presented once every $2.5 \mathrm{sec}$.

After a unit was isolated, its frequency-threshold curve and best frequency were determined audiovisually using tone bursts as stimuli. Afterward, we determined the rate level function (RLF) of the unit to $\mathrm{P}$ alone at a fixed azimuth of $\mathrm{c} 90^{\circ}$. We next determined the responses of the unit to $\mathrm{M}$ alone at a constant level (at $6 \mathrm{~dB}$ above the response threshold of the unit to the best frequency at $\mathrm{c} 90^{\circ}$ ) at various azimuths. The masker loudspeaker was initially positioned at $c 90^{\circ}$ and later moved in random order to $45^{\circ}, 0^{\circ}$, ipsilateral $45^{\circ}\left(\mathrm{i} 45^{\circ}\right)$, and $\mathrm{i} 90^{\circ}$. The response to $\mathrm{M}$ alone served as a reference for the response of unit to $\mathrm{P}+\mathrm{M}$. With the masker at different azimuths, we collected a series of RLFs to $\mathrm{P}+\mathrm{M}$ by changing the probe level systematically from subthreshold to suprathreshold levels in 1-2 $\mathrm{dB}$ increments; these RLFs will be referred to as masked RLFs (MRLFs).

For each cell, we collected the responses of the unit to 10 epochs of a stimulus and calculated the average spike count and SD of it. The detection thresholds for $\mathrm{P}$ alone and for $\mathrm{P}+\mathrm{M}$, were estimated from the RLFs and MRLFs of the unit, respectively, using $d^{\prime}$ analysis from the signal detection theory, taking the firing variance into account (Sakitt, 1973; Jiang et al., 1997; Palmer and Shackleton, 2002). In our preparation, this analysis produced results that were in good agreement $(r>0.96)$ with the threshold estimates based on an increase in firing rate (Ratnam and Feng, 1998; Lin and Feng, 2001). For $d^{\prime}$ analysis, the spike count was converted into $d^{\prime}$ using the following equation:

$$
d^{\prime}=\left(R_{\mathrm{P}+\mathrm{M}}-R_{\mathrm{M}}\right) / \sqrt{S D_{\mathrm{P}+\mathrm{M}} S D_{\mathrm{M}}},
$$

where $R_{\mathrm{P}+\mathrm{M}}$ and $R_{\mathrm{M}}$ are the mean responses to $\mathrm{P}+\mathrm{M}$ and $\mathrm{M}$ alone, and $S D_{\mathrm{P}+\mathrm{M}}$ and $S D_{\mathrm{M}}$ are the $\mathrm{SDs}$ of the respective responses. The detection threshold for $\mathrm{P}+\mathrm{M}$ at a specific azimuth was defined as the lowest probe level at which $d^{\prime}=1$, with the condition that $d^{\prime}$ at the next level was greater than at the threshold (Fig. 2). As shown in the composite raster dot histograms (Fig. 2, right plots), units fired more vigorously at the threshold level than at subthreshold levels, as expected, with evidence of time-locked discharges to the amplitude modulations in the probe at suprathreshold levels. Note that for P alone, there was no masker in the stimulus; thus, for calculation of $d^{\prime}$, we used the spontaneous firing of the unit as $R_{\mathrm{M}}$ and the response of the unit to $\mathrm{P}$ alone as $R_{\mathrm{P}+\mathrm{M}}$.

Because the experimental protocol was very long, to ensure timely completion of the experimental protocol, instead of measuring $R_{\mathrm{M}}$ directly, we used $R_{\mathrm{P}+\mathrm{M}}$ at very low probe levels (subthreshold) to estimate this value. A similar procedure was used in previous binaural masking level difference (BMLD) studies (Jiang et al., 1997; Palmer et al., 2000).

In addition to rate-based $d^{\prime}$ analysis, we also measured the synchronization coefficients from responses to $\mathrm{P}$ alone, $\mathrm{P}+\mathrm{M}$, and $\mathrm{M}$ alone, taking advantage of the differences in the firing synchrony to the signal envelope (Fig. 2). We found that the time-locking data could be used as a second metric for assessing the detection thresholds of a unit for $>60 \%$ of IC neurons. For the rest of IC neurons, however, their response to even $\mathrm{P}$ alone showed little or no statistically significant time locking, yet probe detection was easily determined using rate-based $d^{\prime}$ analysis. Also, for the first population of IC neurons, the cutoff points for the synchronization coefficient (wherein the coefficient was significantly different from the background value) were variable, without a single standard that could be used for all neurons. In light of these findings, we used rate-based $d^{\prime}$ analysis exclusively for determining probe detection.

Masking by noise originating from the same azimuth typically elevated the probe detection threshold of the unit (Ratnam and Feng, 1998; Lin and Feng, 2001). The elevation in threshold, in decibels, therefore indicated the severity of masking. Spatial separation of $\mathrm{P}$ and M typically lowered the probe detection threshold. The decrease in threshold, in decibels, gave an indication of the strength of spatial unmasking.

To test the working hypothesis, we compared the strengths of spatial unmasking for IC neurons before (i.e., control condition) and during (i.e., experimental condition) drug application. Drug application was performed by applying a positive current of 10-30 nA. During continuous drug application, the response of a unit to $\mathrm{P}$ alone was studied at a fixed sound level to observe the progressive increase in response with time. When the drug-induced increase in the firing rate of a unit had saturated, usually after bicuculline had been injected for 15-20 min, we began collection of experimental data by studying the RLF of the unit to $\mathrm{P}$ alone and the MRLFs of the unit to $\mathrm{P}+\mathrm{M}$ at various masker azimuths. After the experimental protocol was completed, bicuculline application was withdrawn. Then, after a waiting period of $\sim 30 \mathrm{~min}$, the experimental protocol was repeated once again, this time with a retention current of $-15 \mathrm{nA}$ to observe the recovery of the control response of the unit. Twotailed Student's $t$ test was used to compare the mean results derived under the control and experimental conditions.

A previous study in the frog IC (Zhang et al., 1999) showed that local application of bicuculline flattens or elevates the response of a unit to interaural level differences. Flattening of this response function is expected to reduce the sensitivity of the unit to sound direction. To determine the extent to which the effect of bicuculline application on spatial unmasking is mediated by a change in the directional sensitivity of a unit, we graphed the directional response function of the unit by plotting the response of the unit to $\mathrm{M}$ alone against sound direction before and during drug application. In the frog IC, the directional response pattern of a unit is predominantly sigmoidal, with a maximal response at $\mathrm{c} 90^{\circ}$ and a progressively weaker response as sound is rotated from c 90 to $190^{\circ}$ (Gooler et al., 1996). As such, we could determine the directional sensitivity of the unit by measuring the response change between the response peak at $\mathrm{c} 90^{\circ}$ and the response trough at $190^{\circ}$ (or at a different azimuth for a few IC neurons). Because bicuculline markedly increases the auditory responses of a unit (albeit nonuniformly across different sound azimuths), the absolute response change between $\mathrm{c} 90$ and $190^{\circ}$ under the experimental condition would overestimate the directional sensitivity of the unit. For this reason, the directional responses of the unit for the two experimental conditions were first normalized before measuring the response change between $\mathrm{c} 90$ and $190^{\circ}$. 
Control
(A) P alone@c $90^{\circ}$
(B) P+ $M @ \mathrm{c} 90^{\circ}$
(C) P+M@ $\mathrm{c} 45^{\circ}$
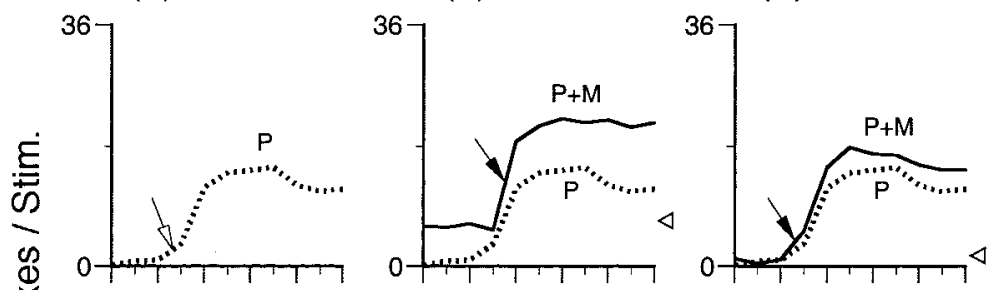

(E) $P+M @ i 45^{\circ}$
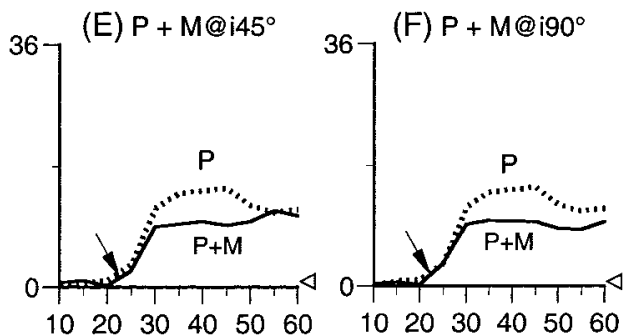

BIC

(H) P alone@c $90^{\circ}$

(l) $\mathrm{P}+\mathrm{M} @ \mathrm{c} 90^{\circ}$

(J) P+M@C45
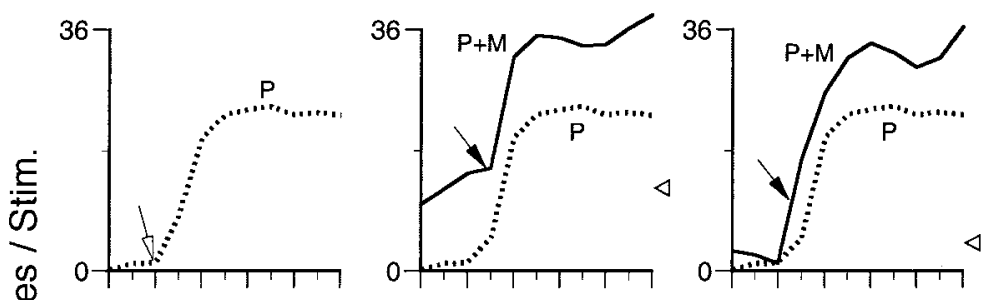

(K) $P+M @ 0^{\circ}$

(L) $P+M @ i 45^{\circ}$

(M) P+M@i $190^{\circ}$

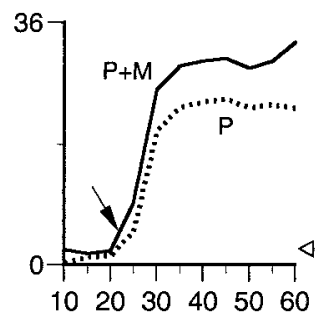

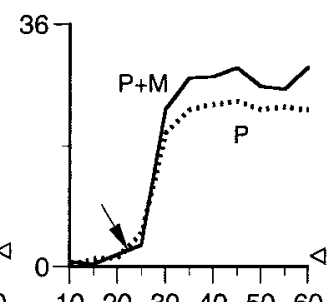

102030405060 Intensity (dB SPL)

\section{(G) Control}

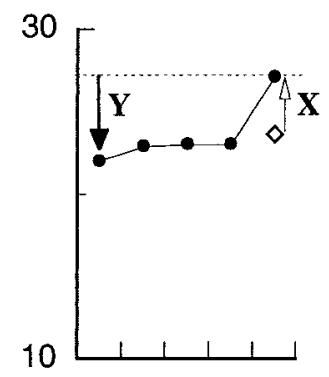

(N) BIC

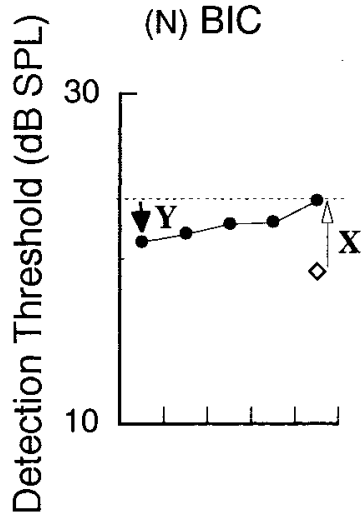

(O) Recovery

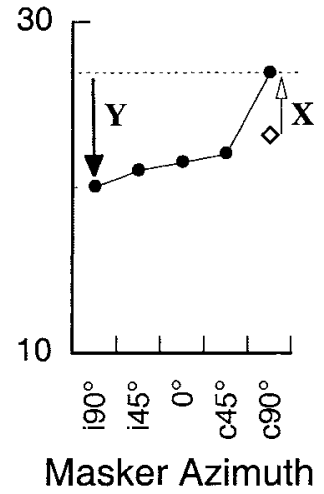

Figure 3. Spatial unmasking response patterns of unit $28-2$ (best frequency, $700 \mathrm{~Hz}$ ). $A$, RLF of the unit to $P$ alone at $c 90^{\circ}$ (this RLF is also shown in $B-F$ as a dotted curve); the open arrow indicates the probe detection threshold to this stimulus as determined by $d^{\prime}$ of $1 . B-F, M R L F s$ to $P+M$ (solid curve) at various masker azimuths; for each plot, the filled arrow indicates the probe detection threshold for $P+M$ (i.e., the sound level for which $d^{\prime}=1$ ). $G$, Change in the probe detection threshold as a result of masking and spatial unmasking (through angular separation of $P$ and $M$ sources) during the control period before drug application; the diamond represents the probe detection threshold for $P$ alone; $X$, amount of threshold elevation attributable to masking; $Y$, maximum masking release attributable to source separation. $H-N$, Response characteristics of the unit during the period when bicuculline was applied iontophoretically $(15 \mathrm{nA}) .0$, Spatial unmasking response pattern of the unit 40 min after withdrawal of bicuculline.

\section{Results}

Complete spatial unmasking data were obtained from 28 IC neurons (and partial data were obtained from 59 IC neurons) under the control and experimental conditions, i.e., before and during drug application. As reported previously (Ratnam and Feng, 1998; Lin and Feng, 2001), the characteristic frequencies (CFs) of IC neurons ranged from 200 to $1700 \mathrm{~Hz}$ and were distributed around three clusters: $200-700,900-1200$, and 1500-1700 Hz. The threshold at the best frequency of a unit from $\mathrm{c} 90^{\circ}$ was distributed over 20-42 dB SPL.
The spatial unmasking response characteristics of a representative IC unit are shown in Figure 3. This unit showed no spontaneous firing during the predrug control period, and the probe was detected at 23.6 dB SPL at which $d^{\prime}=1$ (Fig. $3 A$, open arrow, $G$, diamond). The response of the unit to $\mathrm{P}+\mathrm{M}$ is shown by the MRLF (Fig. 3B, solid line). The MRLF was shifted upward (and also slightly rightward) from the RLF of the unit because of higher background firing, as shown by the response of the unit to $M$ alone (Fig. 3B, open triangle). This shift elevated the probe detection threshold of the unit by $3.5 \mathrm{~dB}$ (shift is shown as $\mathrm{X}$ in Fig. 
(A) $27-1$

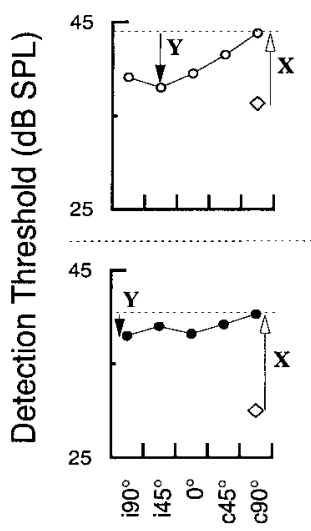

(B) $30-2$
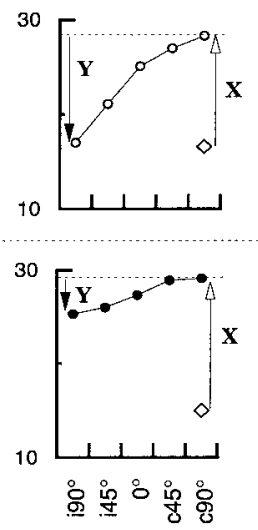

(C) $18-1$
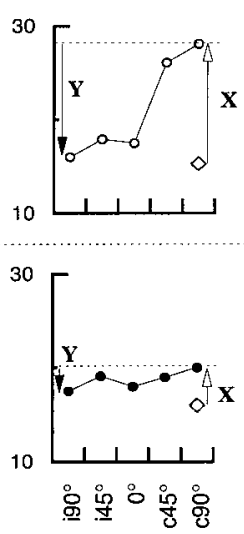

Masker Azimuth
(D) $19-1$

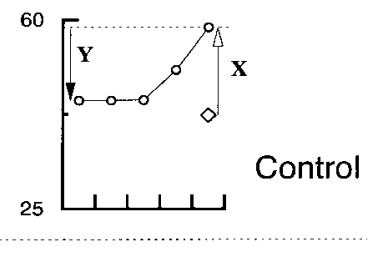

60

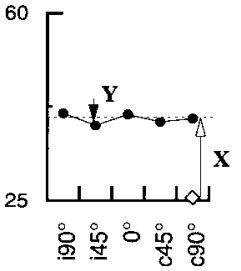

$\mathrm{BIC}$

Figure 4. Spatial unmasking response patterns of four representative IC neurons. See legend to Figure 3 for the different symbols. A, B, Data from two units with graded spatial unmasking patterns under the control (i.e., predrug) and experimental (i.e., when BIC was applied iontophoretically) conditions. C, D, Responses of two units with J-type spatial unmasking patterns under the control condition.

$3 G)$. A change in the masker azimuth to $c 45^{\circ}$ lowered the response of the unit to $\mathrm{M}$ alone (Fig. $3 C$, open triangle) and produced a downward and leftward shift in the MRLF, thereby lowering the probe detection threshold (Fig. 3C, solid arrow). Further spatial separation of probe and masker sources produced only a slight change in the probe detection threshold (Fig. 3G). The maximum masking release of $5.1 \mathrm{~dB}$ (Fig. $3 G, \mathrm{Y}$ ) was obtained with the masker located at $190^{\circ}$.

Drug application increased the probe responses across a wide range of sound levels (Fig. 3, compare $A, H$ ). For this unit, the increase in firing rate lowered the probe detection threshold to 19.2 dB SPL (Fig. 3N, diamond). Under bicuculline, the response of the unit to $\mathrm{M}$ alone at $\mathrm{c} 90^{\circ}$ (Fig. $3 \mathrm{I}$, open triangle) was also more robust (Fig. 3, compare $B, I$ ), thereby elevating its MRLF (Fig. 3I, solid curve). As a result, the probe detection threshold was raised to $23.5 \mathrm{~dB}$ SPL (Fig. $3 \mathrm{~N}, \mathrm{X}$ ). Unlike the control condition, however, this threshold increase was only partially rescued by spatial separation of probe and masker sources (Fig. $3 N$ ). Moving the masker to $\mathrm{c} 45,0$, i 45 , and $190^{\circ}$ lowered the probe detection threshold, but only slightly (Fig. 3J-M). The maximum masking release, obtained with the masker at $190^{\circ}$, was a mere 2.1 $\mathrm{dB}$ (Fig. $3 \mathrm{~N}$ ), which was $3 \mathrm{~dB}$ less than the control condition.

Forty minutes after withdrawal of the drug, the probe responses returned to the levels observed during the predrug period, and the responses to $\mathrm{P}+\mathrm{M}$ at various masker azimuths also closely approximated those shown during the initial control period. Thus, the spatial unmasking pattern of the unit (Fig. 3O) resembled the pattern shown during the control period (Fig. 3G). Specifically, an angular separation of $45^{\circ}$ released the masking effect completely.

Figure 4 shows the spatial unmasking patterns of four other IC units during the control and experimental periods. Unit 27-1 (Fig. 4A) showed a graded spatial unmasking pattern during the control period (top plot). The probe detection threshold was elevated by $7.5 \mathrm{~dB}$ because of masking at $\mathrm{c} 90^{\circ}$ (top plot, X). Spatial separation of $\mathrm{P}$ and $\mathrm{M}$ sources conferred a progressive increase in masking release that was maximal $(5.8 \mathrm{~dB})$ at a masker azimuth of $i 45^{\circ}$ (top plot, Y). Under bicuculline, the masker at $\mathrm{c} 90^{\circ}$ produced a threshold shift of $10.3 \mathrm{~dB}$ (bottom plot, $\mathrm{X}$ ); this was slightly greater than during the control period, but spatial

unmasking was noticeably weaker, with a maximum masking release of $2.3 \mathrm{~dB}$ only (bottom plot, Y).

Similarly, for unit 30-2 (Fig. 4B), the masker at $\mathrm{c} 90^{\circ}$ respectively elevated the probe detection threshold by 11.7 and 14.1 $\mathrm{dB}$ during the control and experimental periods (top, bottom plots, X). The strengths of spatial unmasking in both periods were once again different. The maximum masking release values were $11.3 \mathrm{~dB}$ during the control period and only $3.8 \mathrm{~dB}$ during the experimental period.

Units 18-1 and 19-1 showed J-type spatial unmasking patterns during the control period (Fig. 4C,D). For these units, angular separation of $45-90^{\circ}$ rescued much of the masking effect, with additional spatial separations having little or no further masking release. Drug application weakened the masking release (bottom plots, smaller Y), even though the elevation in threshold attributable to masking was smaller than that of the control period.

The spatial masking and unmasking data from the entire sample $(n=28)$ is summarized in Figures 5 and 6. Masking-induced threshold shifts varied greatly among IC units, irrespective of the best frequency of the unit, during both the control and experimental periods. During the control period, the threshold shift ranged from 1 to $20.9 \mathrm{~dB}$, with a mean $\pm \mathrm{SD}$ of $8.38 \pm 4.95 \mathrm{~dB}$ (Fig. 5A; these data are in agreement with the results of Lin and Feng, 2001). Under bicuculline, the amount of threshold shift could be increased or slightly decreased (Fig. $6 \mathrm{~A}$ ), with a range of -0.5 to $18.5 \mathrm{~dB}$ and a mean of $10.14 \pm 6.52 \mathrm{~dB}$ (Fig. $5 B$ ). These average threshold shifts were not significantly different $(p>$ $0.25)$.

Drug application consistently reduced the strength of masking release (Fig. $6 B$ ). As shown in Figure 5, $C$ and $D$, the maximum masking release during the control period ranged from 3.1 to $14.6 \mathrm{~dB}$, with a mean of $8.57 \pm 3.19 \mathrm{~dB}$ (this agrees well with the result of Lin and Feng, 2001). The maximum masking release was much weaker during the experimental period, with a range of $0.3-9.6 \mathrm{~dB}$ and a mean of $3.94 \pm 2.48 \mathrm{~dB}$. These two means were significantly different $(p<0.0000002)$.

Additionally, for units with J-type spatial unmasking patterns $(n=14$; Fig. $4 C, D)$, the angular separations required for achieving $75 \%$ of the maximum masking release were on average $77^{\circ}$ during the control period and $135^{\circ}$ during the experimental period. These two means were significantly different $(p<0.0003)$. However, for the remaining IC units with graded and complex spatial unmasking patterns, the angular separations required for achieving $75 \%$ of the maximum masking release during the control (i.e., $140^{\circ}$ ) and experimental (i.e., $122^{\circ}$ ) periods were not significantly different $(p>0.1)$.

For five IC units (Fig. 6B, open circles), application of bicuculline had very small effects over the strength of spatial unmasking; spatial unmasking remained robust under the experimental condition, and the maximum masking release was reduced by $<1.5 \mathrm{~dB}$. These neurons presumably have inherited their spatial unmasking properties from lower brainstem neurons and auditory nerve fibers.

To gain further insight into the particular GABAergic processing that was responsible for spatial unmasking, we determined 


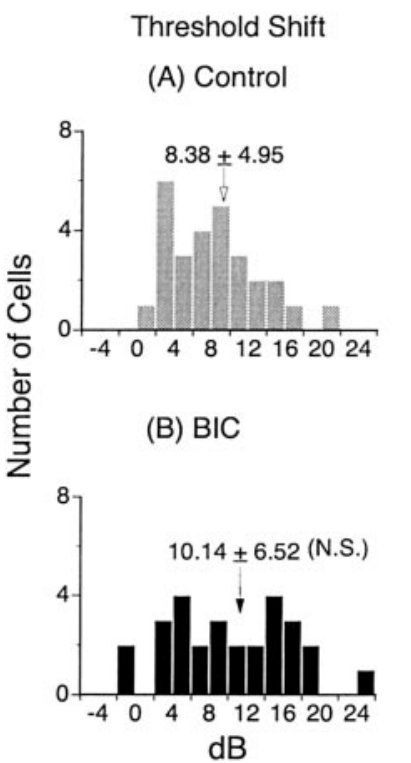

Maximum Masking Release

(C) Control

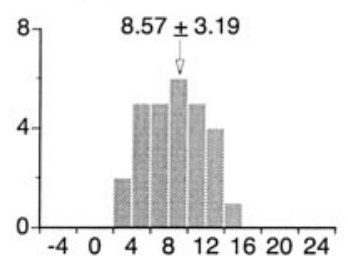

(D) BIC

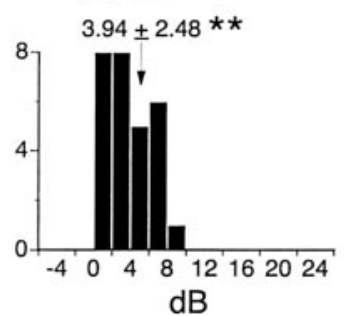

Figure 5. Distributions of threshold shift attributable to masking and the maximum masking release attributable to spatial separation for $28 \mathrm{IC}$ units studied under two different experimental conditions. The mean \pm SD of each data set are shown above each graph. The results of two-tailed Student's $t$ tests between the same data set from two different experimental conditions are shown in $B$ and D. N.S., Not statistically significant. ${ }^{*}$ Statistically significant difference.
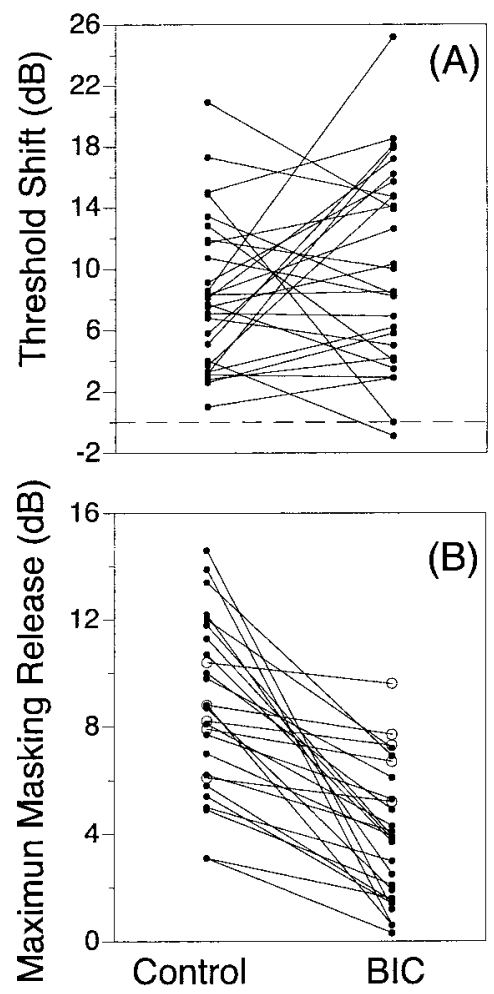

Figure 6. Amount of threshold shift $(A)$ and maximum masking release $(B)$ for each of the $28 \mathrm{IC}$ units under the normal (left side) and experimental (right side) conditions. Open circles in $B$ represent the data from five IC units that were minimally affected by drug application.

the extent to which the sensitivity of a unit to sound direction contributed to spatial unmasking. For this, we plotted the directional responses of IC units to $\mathrm{M}$ alone under the control and experimental conditions. We found that, although bicuculline consistently increased the auditory response of a unit to $\mathrm{M}$ alone,
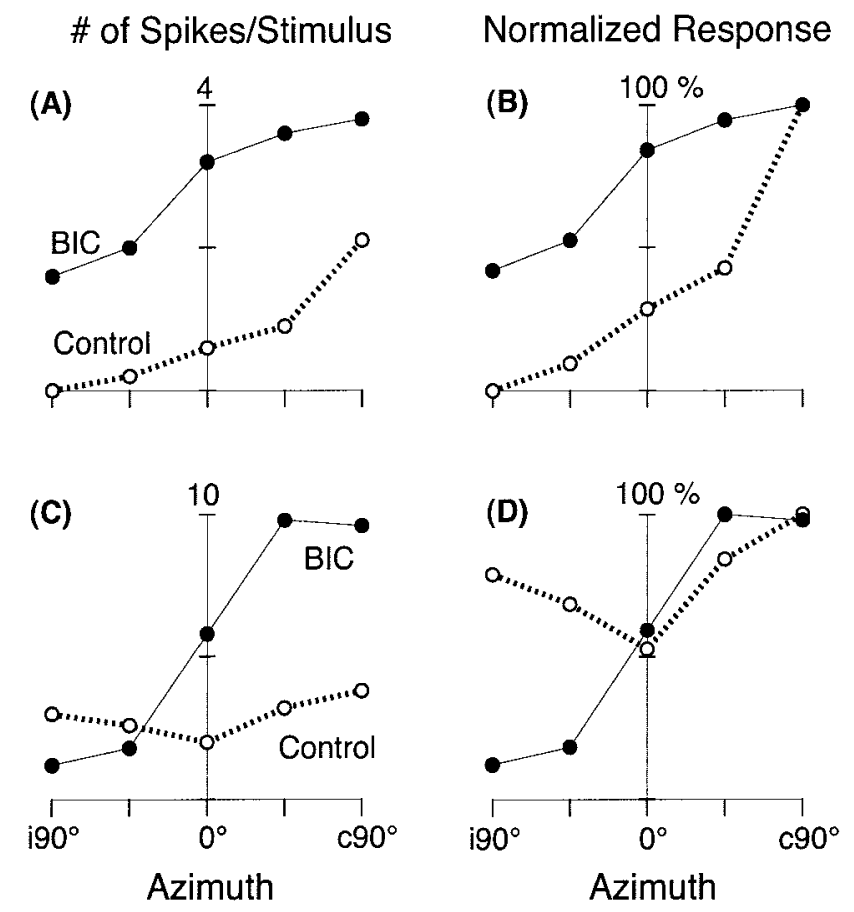

Figure 7. Directional responses of two IC units to $M$ alone at a suprathreshold level during the control (open circle) and experimental (filled circle) conditions. $A, B$, Directional responses from a unit whose directional sensitivity was reduced by application of $B I C$, using the absolute spike count and normalized response (with regard to the maximum spike count for the respective response curve) as a metric. C, D, Similar data from a unit whose directional sensitivity was increased by application of BIC.

its influence over the directional sensitivity of a unit was variable. Figure $7 \mathrm{~A}$ shows a representative effect of bicuculline on the directional response of a unit. This unit showed a sigmoidal directional response pattern, responding maximally to $\mathrm{M}$ alone from $\mathrm{c} 90^{\circ}$, and its response decreased progressively when the masker azimuth was changed toward $i 90^{\circ}$. Bicuculline elevated the responses of the unit at all azimuths, leaving its directional response pattern unchanged; i.e., it remained sigmoidal. However, for this neuron, application of bicuculline reduced its directional sensitivity (Fig. $7 B$ ); the directional sensitivity of the unit was represented by the change in the normalized response between $\mathrm{c} 90$ and i $90^{\circ}$ (see Materials and Methods).

Results from all 28 IC units showed that, during the control period, the percent change in firing rate with an $180^{\circ}$ change in azimuth was on average $90.5 \%$. Under bicuculline, the average percent change was only $68.5 \%$. These averages were significantly different $(p<0.0004)$.

The above population data indicated that a reduction in the strength of spatial unmasking resulting from drug application was likely attributed to a reduction in the directional sensitivity of a unit. Analysis of individual data (Fig. 8), however, showed that such a correlation was applicable to only a population of IC units. Specifically, for 15 of the 28 units studied (bottom plot bounded by dashed, dotted oval), bicuculline gave rise to a wide range of reduction in directionality (range, 10-90\%). For these, a large reduction in the directionality is accompanied by a marked weakening of spatial unmasking. For 10 cells (bounded by dotted oval), however, bicuculline produced only a small change $(<20 \%)$ in directionality, yet most of these cells showed a noticeable reduction in the strength of spatial unmasking. For two IC neurons (Fig. 8, top half), bicuculline actually increased the directionally sensitivity of the unit, i.e., by 28 and $67 \%$, respectively. 


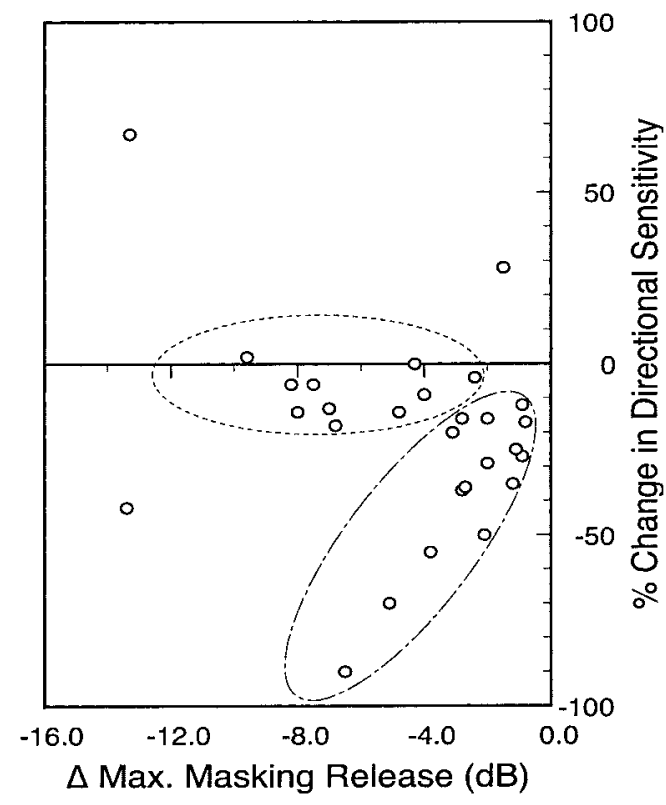

Figure 8. Effects of drug application on the directional sensitivity and spatial unmasking of IC units. Directional sensitivity was measured by the total percent change in normalized directional responses between $\mathrm{c} 90$ and $i 90^{\circ}$ (see Fig. 7). The $x$-axis shows the difference in the maximum masking release (see Figs. 3,4,Y) between the control and experimental conditions, with negative values indicating a decrease in masking release resulting from drug application. The $y$-axis shows the difference in the percent change in the directional sensitivity of the unit between the control and experimental conditions; negative and positive values indicate, respectively, a decrease and increase in directional sensitivity resulting from drug application.

As shown for one of these cells (Fig. 7C), the increase in directional sensitivity was attributable to a pronounced increase in the response of the unit at $c 45-\mathrm{c} 90^{\circ}$ and a decrease in response at $\mathrm{i} 45-\mathrm{i} 90^{\circ}$. Despite the increase in directional sensitivity, the strength of spatial unmasking for these two neurons was either unchanged or totally abolished.

\section{Discussion}

The present study shows that application of bicuculline significantly reduces the strength of spatial unmasking in the IC such that even a large angular separation of probe and masker sources produces only a weak masking release. Under bicuculline, the spatial unmasking patterns of IC neurons are mostly flat or slowly graded, and the maximum masking release occurs primarily with a large spatial separation of $135-180^{\circ}$, similar to the patterns observed at the auditory periphery (Lin and Feng, 2001). Specifically, the average maximum masking release for IC units under the control condition is $8.38 \pm 4.95 \mathrm{~dB}$, but under the experimental condition, it is reduced to $3.94 \pm 2.48 \mathrm{~dB}$, approximating that for the periphery $(2.90 \pm 2.79 \mathrm{~dB}$; Lin and Feng, 2001). The averages for the IC under the experimental condition and for the periphery are not significantly different $(p>0.12)$. The above results suggest that GABAergic inhibitory interactions within the IC play an important role in enhancing spatial unmasking.

Whereas bicuculline weakens spatial unmasking of IC units, angular separation still produces some masking release. The weak masking release that remains is likely attributed to acoustic shadowing of the head, as observed in the auditory periphery (Lin and Feng, 2001). In the periphery, head shadowing contributes to spatial unmasking; namely, moving the masker source away from the probe source weakens the masker energy relative to the probe at the stimulated ear. Thus, similar to mammals, the frog audi- tory system relies on both peripheral and central processing to unmask a signal spatially.

For five IC neurons, the drug-induced change in the strength of spatial unmasking was small, i.e., $\leq 1.5 \mathrm{~dB}$ (Fig. $6 B$, open circle). For these units, central processing occurring at lower brainstem nuclei and the auditory nerve are likely responsible for spatial unmasking.

Previous studies have shown that GABA plays many different roles in auditory processing in the IC, monaural and binaural (Faingold et al., 1991; Fujita and Konishi, 1991; Yang et al., 1992; Vater et al., 1992; Park and Pollak, 1993, 1994; Casseday et al., 1994; Klug et al., 1995; Fuzessery and Hall, 1996; Palombi and Caspary, 1996; Sanes et al., 1998; Jen and Feng, 1999; Zhang et al., 1999; Zhou and Jen, 2000). These studies have shown that application of bicuculline changes the temporal discharge pattern and firing latency of a unit, broadens the frequency tuning and spatial receptive field of the unit, abolishes duration selectivity, alters the response profile to interaural time differences, and abolishes or weakens the sensitivity to interaural level differences. Of these, the role of GABA in binaural processing of time and level differences is likely most important for spatial unmasking. This tenet is compatible with the findings from psychophysical studies in humans (Yost, 1997) and ferrets (Moore et al., 1999), showing that binaural processing is important for spatial unmasking.

In humans, psychophysical studies are based mostly on dichotic experiments and use the BMLD paradigm. A dominant model for explaining the BMLD results is a running crosscorrelation model of binaural interaction that includes a network of coincidence detectors used for processing the interaural time difference cue (Colburn, 1996). Physiological studies of BMLD in mammals using dichotic stimulations have conferred results that support such model (for review, see Palmer and Shackleton, 2002). At present, the role of processing of the interaural intensity difference in spatial unmasking has not been adequately explored. Also, because BMLD experiments typically use a lowfrequency probe with a single interaural phase-time difference, it would be a stretch to generalize the BMLD data to real-world listening conditions (e.g., to free-field spatial unmasking of broadband signals).

For frogs, neurons in the superior olivary nucleus and the auditory midbrain show poor phase-locking ability (Condon et al., 1995). Thus, running cross-correlation of binaural time differences unlikely plays a role in directional coding and in spatial unmasking. In the frog IC, the dominant binaural interaction pattern is binaural inhibition that is involved in processing the interaural level difference cue (Epping and Eggermont, 1985; Gooler et al., 1996). Zhang et al. (1999) further showed that application of bicuculline in the frog IC abolishes binaural inhibition, suggesting that GABAergic synaptic interactions taking place within the IC are mostly responsible for binaural inhibition. Taken together, those data, along with results from this study, suggest that, for frogs, binaural inhibition that is important for shaping the directional sensitivity of IC neurons is primarily responsible for spatial unmasking. Namely, application of bicuculline abolishes binaural inhibition that in turn reduces the sensitivity to interaural level differences and thus to sound direction. As a result, spatial unmasking is compromised, such that even large angular separations produce little masking release. Results from the pooled data in the present study are supportive of this interpretation, because application of bicuculline reduces the directional sensitivity of, and weakens spatial unmasking in, the majority of IC units.

We note, however, that there are exceptions to the general 
trend described above. For one-third of IC units, bicuculline has a small to moderate effect on the directional sensitivity of the units, yet their strength of spatial unmasking is markedly compromised (Fig. 8). The strongest counterexample is from one IC unit that becomes directionally more sensitive under the experimental condition (Fig. 7C,D), but its strength of spatial unmasking is essentially completely abolished. Clearly, for these IC units, different GABAergic-based processing is responsible for the observed weakening of spatial unmasking.

In crickets and katydids, the $\Omega$ neurons in the nerve cord have been shown to be responsible for spatial unmasking (Pollack, 1988); these neurons display masking release by directional hearing. However, Römer and Krusch (2000) later showed that such neurons also exhibit "selective attention" to concurrent sounds originating from the same direction. Specifically, they showed that $\Omega$ neurons can encode the probe signal that is slightly more intense (by as little as $2 \mathrm{~dB}$ ) than a competing sound, and this effect persists when the auditory nerve on the contralateral side is severed. Thus, encoding of the weaker sound need not involve binaural processing,

It is possible that a similar attention-based mechanism operates in the frog CNS and that such selective response to the mating trill of the species is independent of binaural processing but dependent on GABAergic interactions nonetheless. We speculate that although bicuculline increases IC unit responses to acoustic stimuli in general, it may abolish the response selectivity of the unit to the mating trill of the species, at least for a subset of IC units. There are two indirect lines of evidence that support this tenet. First, Klug et al. (2002) showed in the free-tailed bat's IC that unit selectivity to species-specific calls is primarily attributed to GABAergic inhibition taking place locally. Second, bicuculline abolishes the duration selectivity of the unit in the bat IC (Jen and Feng, 1999), and duration selectivity has been shown to contribute to the response selectivity of the unit to the frog's mating trill (Feng et al., 1990). Although the above evidence is suggestive, whether the frog's auditory system possesses a similar GABAergic-based selective mechanism requires direct empirical validation. The present data from responses to a trill and noise alone are inadequate for testing the above hypothesis.

At present, the actual binaural inhibitory circuitry responsible for spatial unmasking in the frog IC is unclear. It is unknown whether the circuit involves ascending, descending, or commissural afferents or local interactions. As mentioned earlier, many neurons in the frog caudal brainstem nuclei exhibit binaural facilitation and binaural inhibition (Feng and Capranica, 1976, 1978; Zakon and Capranica, 1981). Binaural processing at lower levels likely contributes to spatial unmasking, as suggested by the data from some of the IC neurons in this study. It seems that such processing is repeated at the IC, presumably to ensure effective detection of a signal in noise at all levels. The roles of descending inputs also cannot be ruled out. The frog IC receives descending afferents (Feng and Lin, 1991), and when descending inputs are stimulated, IC neurons display long-lasting inhibition (Endepols and Walkowiak, 2001).

For this study, the probe was stationed at $\mathrm{c} 90^{\circ}$, which represents the best azimuth for most IC neurons, but frogs likely can tune to listen to sound from various directions during their acoustic interactions, from the lateral as well as frontal sound fields. The benefit of central processing to spatial unmasking for sound located in the frontal field may be different from what is reported in this study and needs to be determined empirically. At this time, it is also unclear whether the strength of spatial unmasking in the IC varies with sound direction. Previously, Rat- nam and Feng (1998) studied spatial unmasking in the frog IC for a probe $(20 \mathrm{~Hz}$ sinusoidally amplitude-modulated tone at the $\mathrm{CF}$ of the unit) located at $0^{\circ}$ and found the strength of spatial unmasking to be not as robust as the control condition reported herein. This difference, however, may be attributed to a difference in either the probe signal or the probe azimuth. Future research (using an identical probe and masker) is necessary for resolving this question.

\section{References}

Bronkhorst AW, Plomp R (1988) The effect of head-induced interaural time and level differences on speech intelligibility in noise. J Acoust Soc Am 83:1508-1516.

Casseday JH, Ehrlich D, Covey E (1994) Neural tuning for sound duration: role of inhibitory mechanisms in the inferior colliculus. Science 264:847-850.

Colburn HS (1996) Computational models of binaural processing. In: Auditory computation (Hawkins HL, McMullen TA, Popper AN, Fay RR, eds), pp 332-400. New York: Springer.

Condon CJ, Chang SH, Feng AS (1995) Classification of the temporal discharge patterns of single auditory neurons in the frog superior olivary nucleus. Hear Res 83:190-202.

Dent ML, Dooling RJ, Larsen ON (1997) Free-field binaural unmasking in budgerigars (Melopsittacus undulatus). Behav Neurosci 111:590-598.

Endepols H, Walkowiak W (2001) Integration of ascending and descending inputs in the auditory midbrain of anurans. J Comp Physiol [A] 186:1119-1133.

Epping WJM, Eggermont JJ (1985) Relation of binaural interaction and spectro-temporal characteristics in the auditory midbrain of the grassfrog. Hear Res 19:15-28.

Faingold CL, Boersma-Anderson CA, Caspary DM (1991) Involvement of GABA in acoustically evoked inhibition in inferior colliculus. Hear Res 52:201-216.

Feng AS, Capranica RR (1976) Sound localization in anurans. I. Evidence of binaural interaction in dorsal medullary nucleus of bullfrogs (Rana catesbeiana). J Neurophysiol 39:871-881.

Feng AS, Capranica RR (1978) Sound localization in anurans. II. Binaural interaction in superior olivary nucleus of the green tree frog (Hyla cinerea). J Neurophysiol 41:43-54.

Feng AS, Lin WY (1991) Differential innervation patterns of individual divisions of frog auditory midbrain (torus semicircularis). J Comp Neurol 306:613-620.

Feng AS, Hall JC, Gooler DM (1990) Neural basis of sound pattern recognition in anurans. Prog Neurobiol 34:313-329.

Fujita I, Konishi M (1991) The role of GABAergic inhibition in processing of interaural time difference in the owl's auditory system. J Neurosci 11:722-739.

Fuzessery ZM, Hall JC (1996) Role of GABA in shaping frequency tuning and creating FM sweep selectivity in the inferior colliculus. J Neurophysiol 76:1059-1073.

Gerhardt HC (1988) Acoustic properties used in call recognition by frogs and toads. In: The evolution of the amphibian auditory system (Fritzsch B, Ryan MJ, Wilczynski W, Hetherington TE, Walkowiak W, eds), pp 455-483. New York: Wiley.

Gilkey RH, Good MD (1995) Sound localization in noise: the effect of signal to noise ratio. J Acoust Soc Am 99:1108-1117.

Gooler DM, Xu JH, Feng AS (1996) Binaural inhibition is important in shaping the free-field frequency selectivity of neurons in the inferior colliculus. J Neurophysiol 76:2580-2594.

Hine JE, Martin RL, Moore DR (1994) Free-field binaural unmasking in ferrets. Behav Neurosci 108:196-205.

Jen PHS, Feng RB (1999) Bicuculline application affects discharge pattern and pulse-duration tuning characteristics of bat inferior collicular neurons. J Comp Physiol [A] 184:185-194.

Jiang D, McAlpine D, Palmer AR (1997) Detectability index measures of binaural masking level difference across populations of inferior colliculus neurons. J Neurosci 17:9331-9339.

Klug A, Park TJ, Pollak GD (1995) Glycine and GABA influence binaural processing in the inferior colliculus of the mustache bat. J Neurophysiol 74:1701-1713. 
Klug A, Bauer EE, Hanson JT, Hurley L, Meitzen J, Pollak GD (2002) Response selectivity for species-specific calls in the inferior colliculus of Mexican free-tailed bats is generated by inhibition. J Neurophysiol 88:1941-1954.

Lin WY, Feng AS (2001) Free-field unmasking response characteristics of frog auditory nerve fibers: comparison with the responses of midbrain auditory neurons. J Comp Physiol [A] 187:699-712.

Moore DR, Hine JE, Jiang ZD, Matsuda H, Parsons CH, King AJ (1999) Conductive hearing loss produces a reversible binaural hearing impairment. J Neurosci 19:8704-8711.

Palmer AR, Shackleton TM (2002) The physiological basis of the binaural masking level difference. Acta Acustica 3:312-319.

Palmer AR, Jiang D, McAlpine D (2000) Neural responses in the inferior colliculus to binaural masking level differences created by inverting the noise in one ear. J Neurophysiol 84:844-852.

Palombi PS, Caspary DM (1996) GABA inputs control discharge rate primarily within frequency receptive fields of inferior calculus neurons. J Neurophysiol 75:2211-2219.

Park TJ, Pollak GD (1993) GABA shapes sensitivity to interaural intensity disparities in the mustache bat inferior colliculus: implications for encoding sound location. J Neurosci 13:2050-2067.

Park TJ, Pollak GD (1994) Azimuthal receptive fields are shaped by GABAergic inhibition in the inferior colliculus of the mustache bat. J Neurophysiol 72:1080-1102.

Pollack GS (1988) Selective attention in an insect auditory neuron. J Neurosci 8:2635-2639.

Ratnam R, Feng AS (1998) Detection of auditory signals by frog inferior colliculus neurons in the presence of spatially separated noise. J Neurophysiol 80:2848-2859.

Römer H, Krusch M (2000) A gain control mechanism for processing of chorus sounds in the afferent auditory pathway of the bushcrickets Tet- tigonia viridissima (Orthoptera: Tettigoniidae). J Comp Physiol [A] 186:181-191.

Saberi K, Dostal L, Sadralodabai T, Bull V, Perrott DR (1991) Free-field release from masking. J Acoust Soc Am 90:1335-1370.

Sakitt B (1973) Indices of discriminability. Nature 241:133-134.

Sanes DH, Malone BJ, Semple MN (1998) Role of synaptic inhibition in processing of dynamic binaural level stimuli. J Neurosci 18:794-803.

Schwartz JJ, Gerhardt HC (1995) Directionality of the auditory system and call pattern recognition during acoustic interference in the gray tree frog Hyla versicolor. Audit Neurosci 1:195-206.

Vater M, Habbicht H, Kossl M, Grothe B (1992) The functional role of GABA and glycine in monaural and binaural processing in the inferior colliculus of horseshoe bats. J Comp Physiol [A] 171:541-553.

Wells KD (1988) The effect of social interactions on anuran vocal behavior. In: The evolution of the amphibian auditory system (Fritzsch B, Ryan MJ, Wilczynski W, Hetherington TE, Walkowiak W, eds), pp 433-454. New York: Wiley.

Yang L, Pollak GD, Resler C (1992) GABAergic circuits sharpen tuning curves and modify response properties in the mustache bat inferior colliculus. J Neurophysiol 68:1760-1774.

Yost WA (1997) The cocktail party problem: forty years later. In: Binaural and spatial hearing in real and virtual environments (Gilkey RH, Anderson TR, eds), pp 329-347. Mahwah, NJ: Erlbaum.

Zakon H, Capranica RR (1981) Reformation of organized connections in the auditory system after regeneration of the eighth nerve. Science 213:242-244.

Zhang HM, Xu JH, Feng AS (1999) Effects of GABA-mediated inhibition on direction-dependent frequency tuning in the frog inferior colliculus. J Comp Physiol [A] 184:85-98.

Zhou XM, Jen PHS (2000) Neural inhibition sharpens auditory spatial selectivity of bat inferior collicular neurons. J Comp Physiol [A] 186: 389-398. 